\title{
Mise en oeuvre de la pédagogie de projet afin d'améliorer la production orale des apprenants de français de la Licence en Langues Étrangères à l'Universidad Pedagógica y Tecnológica de Colombia*
}

Implementación de la pedagogía de trabajo por proyectos para el mejoramiento de la producción oral de los estudiantes de francés de la Licenciatura en Lenguas Extranjeras de la Universidad Pedagógica y Tecnológica de Colombia

MISAEL FONSECA AYALA* misaelfonseca.ayala@gmail

\footnotetext{
* Este es un artículo de reflexión resultante de una investigación enmarcada en la línea Pedagogía del Lenguaje.

** Licenciado en Lenguas Extranjeras, Magíster en Letras, Lenguas y Comunicación con énfasis en Lenguas, Cultura e Interculturalidad, especialidad Inglés-Español, Universidad Lille 3, Francia. Magíster en Ciencias Humanas y Sociales, énfasis Ciencias del Lenguaje, especialidad en Didáctica de Lenguas y Culturas, Universidad Lille 3, Francia. Docente Ocasional de Tiempo Completo de la Universidad Pedagógica y Tecnológica de Colombia.
} 


\section{Résumé}

Cet article présente une analyse de l'enseignement du français, spécifiquement dans l'enseignement de la production orale à travers la méthodologie de travail par projets avec des élèves de quatrième semestre du programme des Langues Étrangères de la Universidad Pedagógica y Tecnológica de Colombia.

Mots clés: Pédagogie de projet, français langue étrangère, production orale, compétence linguistique.

\section{Abstract}

This article presents an analysis of French language teaching, more precisely aimed at oral production through project work methodology, with fourth semester students of the Foreign Languages program at Universidad Pedagógica y Tecnológica de Colombia.

Key words: Project pedagogy, French as a foreign language, oral production, linguistic competence.

\section{Resumen}

Este artículo presenta un análisis de la enseñanza del francés, más específicamente en la enseñanza de la producción oral a través de la metodología de trabajo por proyectos, con estudiantes de cuarto semestre del programa de Lenguas Extranjeras de la Universidad Pedagógica y Tecnológica de Colombia.

Palabras clave: pedagogía por proyectos, francés como lengua extranjera, producción oral, competencia lingüística. 


\section{Introduction}

L'enseignement du français en Colombie est devenu l'une des priorités grâce à la gestion faite par le gouvernement français, lequel a prôné pour un retour de cette langue dans les écoles et les institutions publiques et privées du pays. C'est grâce à cela que cette langue est actuellement considérée comme deuxième langue étrangère dans la plupart des écoles publiques, privées, lycées et universités de Colombie. Cela permet notamment au public souhaitant l'apprendre d'accéder à une formation et ainsi de favoriser sa diffusion.

C'est à partir du constat et des problèmes rencontrés concernant la production orale qu'on s'est plongé dans un travail dont le but était d'améliorer cette compétence avec les étudiants de quatrième semestre (1ème semestre 2012) de la Licence en Langues Étrangères à l'Universidad Pedagógica et Tecnológica de Colombia car cette institution offre une formation en Langues, et préconise davantage l'enseignement du français à de jeunes adultes destinés à devenir des formateurs dans les écoles primaires. C'est donc grâce à ce public et après avoir dépouillés les enquêtes et les entretiens faits auprès de quelques professeurs et quelques étudiants qu'on a repéré la problématique surtout liée au fait que trop peu de temps soit consacrée à la compétence mentionnée au préalable en classe de français. Après cela, on s'est penché sur un travail ayant comme objectif d'utiliser les principes de la pédagogie du projet en classe de FLE afin de pallier les besoins des apprenants.

\section{L'enseignement du français au sein de la licence en langues étrangères: problématique rencontrée}

Suite aux 20 enquêtes et aux 4 entretiens menés auprès des étudiants et des professeurs, on a constaté certains problèmes liés à la production orale, surtout lorsqu'on demandait aux étudiants de communiquer, de réagir à des situations préalablement établies et de créer des dialogues. Par ailleurs, les problèmes les plus significatifs étaient le manque d'interaction et le peu de projets consacrés pour améliorer la production orale des apprenants. On a remarqué suite aux entretiens que les étudiants ne travaillaient que dans la salle de classe 
et n'envisageaient pas de projets. Ces derniers pourraient permettre d'améliorer certaines compétences linguistiques chez les étudiants de français, qui auraient été laissées de côté. Bien que l'apprentissage des langues en Amérique et notamment en Colombie ne fonctionne pas de la même manière qu'en Europe, car les étudiants n'ont pas les mêmes avantages en termes d'échanges linguistiques, de temps, d'argent et de distance entre les pays, on devrait motiver les étudiants à travailler beaucoup plus en dehors de la salle de classe pour ainsi pouvoir aborder l'enseignement de la production orale du français autrement.

\section{Motivations pour entamer les projets au sein de la Licence}

Le but des projets était avant tout de pallier la problématique mise en évidence à l'Universidad Pedagógica y Tecnológica de Colombia et notamment dans la Licence en Langues Étrangères concernant la production orale des étudiants de français. Ces projets professionnels visaient aussi al développer trois projets dont l'objectif principal était de motiver les étudiants à communiquer en français en dehors de la salle de classe.

Ces projets ont été créés pour susciter l'intérêt des apprenants et les motiver à̀ communiquer en langue française. Ceux-ci avaient pour objectif de les aider à se débarrasser de la peur de communiquer, car beaucoup d'entre eux avaient des appréhensions lorsqu'ils devaient faire face al̀ une situation réelle de communication par exemple: commander un repas, faire un tour dans la ville, chercher un emploi etc.

Par ailleurs, les projets ont été développés lors des quatre mois de stage au sein de l'Université et principalement de l'École de Langues dans le but d'amener les apprenants vers la recherche de stratégies méthodologiques pour surmonter la peur de parler en public en utilisant la langue française, ainsi que pour les aider à améliorer le niveau linguistique.

Savignon (1990) signale «qu'il est important de créer et de mettre en place des programmes et des méthodologies qui favorisent le développement d'une compétence fonctionnelle en langue étrangère par la participation des apprenants aux situations de communication» (p. 210); c'est pourquoi en tenant compte de cette opinion, la création d'une émission de radio a été entamée pour éveiller la curiosité et le goût des étudiants à communiquer. Ensuite des reportages ont été appliqués afin de faire interagir les étudiants en dehors de la salle de classe tout en utilisant des outils tels que: la langue française et aussi le multimédia; et finalement, un journal télévisé a été réalisé avec les apprenants pour achever les activités et ainsi donner lieu à̀ l'analyse de l'impact des projets et l'amélioration de la production orale dans le groupe.

\section{2}


Oxford (1990) estime que «le développement d'une compétence communicative demande l'interaction réaliste entre apprenants qui utilisent un langage porteur de sens, dans un contexte identifiei» (p. 8). C'est pour cela que ces projets cherchaient à définir des objectifs adaptés aux apprenants, favorisant ainsi les rencontres multiples autour de la langue. Le but est ici d'inciter les étudiants à manipuler la langue, produire de l'information et devenir créatifs.

Il est important de remarquer aussi que l'utilisation des ressources authentiques tels que: des documents qui n'ont pas été conçus à l'origine pour un cours de langue, par exemple: un article de presse, un extrait d'une émission de radio ou de film, une photo, une brochure ou un souvenir de vacances, ce qui a joué un rôle capital dans l'élaboration de ces projets puisque ceux-ci ont favorisé le centrage de l'enseignement sur «les fonctions ou actes de parole» (savoir-faire de type commander un repas, et se confronter à des situations de la vie quotidienne) (Cité par Bérard 1991). Par ailleurs, ces dernières préconisent des situations de communication authentiques donnant une solution aux besoins des apprenants (p. 48).

\section{La pédagogie du projet en lien avec la production Orale}

La pédagogie du projet favorise aussi l'appropriation d'un projet par le groupe, puis par l'individu, «grâce à des moyens simples: un choix des thématiques et des méthodes laissées aux participants; une dynamique de groupe efficace et une valorisation du travail effectueì (Toffoli,2007, p. 48). C'est ainsi qu'al travers la dynamique de travail par projets, on peut arriver al intégrer des multiples contenus et des compétences. Ce dernier constat a été remarqué lors de la mise en œuvre des projets puisqu'on a pu véhiculer d'autres compétences, non seulement linguistiques mais aussi interdisciplinaires.

Ainsi, grâce al̀ l'intégration des contenus dans la pédagogie du projet, des projets ont été créés en tenant compte de la production orale car selon l'exploration, il m'a semblé important de faire interagir et socialiser l'ensemble des apprenants avec une forte utilisation du français dans les échanges et aussi d'innombrables occasions pour s'entraîner à l'oral.

Mais travailler la production orale demande des connaissances grammaticales, lexicales et phonétiques. Selon Canale \& Swain (1980) la communication comporte des compétences grammaticales, sociolinguistiques, conversationnelles et stratégiques. Dans le cas de projets, ceux-ci cherchaient à aider les apprenants à favoriser les rencontres autour de la langue française, surtout concernant l'entraînement à̀ la compréhension, créer des occasions pour imiter, répéter, comprendre les aspects systématiques de la langue, manipuler les situations de communication, produire et être créatif et insister sur l'authenticité de la communication. 
Par ailleurs, les projets ont été édifiés afin de développer certains critères basés sur la théorie de l'approche communicative qui préconise:

1. La primauté du sens par rapport à la forme

2. Des interactions réalistes

3. Une préparation à̀ la vie réelle

4. Un langage contextualiseì et porteur de sens

5. Des ressources authentiques

\section{Résultats obtenus grâce aux projets}

La production orale a été l'un des points les plus importants dans ce projet. Elle a joueì un rôle capital puisque les projets ont été élaborés en tenant compte des rencontres multiples autour de la langue française, ainsi que de sa manipulation pour arriver à̀ mieux communiquer et avec cela à améliorer le niveau linguistique.

Depuis la naissance des projets, un manque d'interaction et de communication a été constaté en langue française chez ces apprenants. Cela provoquait des ennuis dans la salle de classe et une démotivation générale. C'est pourquoi, la pédagogie de projet les a aidés à progresser et a pu ainsi les guider vers l'acquisition des stratégies pour améliorer leur production orale. Ces dernières consistaient à parler avec une personne en qui l'apprenant n'avait pas confiance, à mener une conversation par soi même et enfin à être à l'écoute pour pouvoir poser de questions et participer aux interactions. Pour y arriver, quelques exercices ont été appliqués, issus de différentes méthodes telles que: Réussir le Niveau B1 du CIEP, Agenda 3, A propos, Alter ego 3 et Echo 3 dans le but de dédramatiser la parole et de minimiser la timidité qu'ils avaient de parler en langue française.

L'ensemble des apprenants ont travailleì d'abord par rapport à̀ quelques situations contextualisées lors desquelles ils devaient toujours jouer un rôle pour «expliquer une situation, donner son opinion, se justifier, insister, être d'accord avec l'autre personne, dire la vérité, exprimer des regrets...». Ensuite, ils devaient jouer des scènes un peu plus complexes telles que: «En France, vous retirez de l'argent al̀ un distributeur automatique de billets avec votre carte bancaire. Mais vous vous rendez compte que l'appareil vous a donneì 10 euros de moins que la somme demandée. Vous tentez d'obtenir le remboursement des 10 euros auprès de l'employé de l'agence». La scène citée ci-dessus leur avait permis d'approfondir leurs connaissances, de s'imprégner de la culture et aussi de chercher le

\section{4}


lexique nécessaire pour pouvoir s'exprimer dans de telles situations. Cependant, la communication dans certains cas était très limitée al̀ cause du manque de vocabulaire, cela a été remarquè du aux longues pauses qu'ils faisaient lors du discours et al̀ la demande continue d'arrêter la communication.

Exemple: situation de communication 1.

$\mathrm{X}$ : Il ne faut pas exagérer + Nous $\backslash \backslash$ eitions entoureìs des couturiers et des top models les plus branchés et eilè̀gants et c'était qu'ils portaient ses propres crè̀ations (1.2) en plus je suis habillè̀ avec une robe dessineì par Christian Dior qui eitait al̀ côté de nous

Attendez -moi un moment professeur, j'ai oublieì les mots sil.

1 minute aprè̀s:

C: Je ne crois pas que Christian Dior l'aurait dessineìe. Bon, «:» comme je te disais, tout eitait trel̀s mauvais (1.5) Est-ce que tu n'as pas vu les model̀les. Elles eitaient trel̀s grosses et avec ses dessins, semblaient comme des mannequins sortis d'un cirque. AÌ mon avis/ meme pas les clowns ne s'habilleraient pas comme cela.

Or, une primauté du sens a été remarquée par rapport à̀ la forme car ils n'ont pas formuleì de phrases bien faites et leur vocabulaire n'était pas très étendu. Cependant, tout ce qu'ils disaient était compris et ils étaient capables de faire passer le message.

Malgré les interactions réalistes qu'ils ont réalisées en classe, il y avait un grand nombre d'apprenants qui faisaient beaucoup d'erreurs concernant la syntaxe comme par exemple: «Rapelles-Tu,Je suisle Directeur du magazine "lavie moderne", Qui est trè̀s du influence dans milieu de la mode. Donc, al partir de maintenant, Tu seras mon avis sur la fac'on dont je m'babille. Bien, ensuite, Je serai brancheì. Ils avaient du mal à faire la différence entre phonème et graphie Par exemple : enfain ou lieu de dire enfin et joune au lieu de dire jeune . On remarque aussi des problèmes de conjugaison au présent et au passeì composeì tels que: tu aimez, tu es aimeì de mon deifileì, je penseì. Par ailleurs, il y a aussi des difficultés avec le genre: ton façon au lieu de ta façon.

En outre, il faut indiquer que suite aux observations participatives, on a remarqué que les étudiants apprenaient beaucoup de vocabulaire et d'énoncés par cœur. Cependant, ils ne pouvaient pas soutenir une conversation. Une absence d'expression et de flexibilité a été 
repérée dans le langage; tout cela est dû à un exercice où ils devaient s'exprimer par rapport aux problèmes à surmonter lorsqu'on quitte le foyer maternel; La situation citée au préalable a évidemment aidé puisque ce qui était recherché au travers des projets, c'était de leur donner des outils pour développer un peu plus la spontanéité et avec cela, réussir à ce qu'ils s'engagent dans une conversation ou un simple dialogue. On peut dire qu'ils réfléchissaient beaucoup pour chercher des mots et des expressions afin d'assurer la communication et les mises en situation ont constitueì une vraie préparation pour des interactions réalistes.

Ainsi, aprè̀s avoir travailleì pendant 4 semaines en créant des dialogues et des conversations toutes faites, tous des apprenants se sont lancés dans la création d'une émission de radio, des reportages et d'un journal télévisé, lesquels allaient leur donner une certaine confiance pour parler en français et avec cela pouvoir surmonter les problèmes de communication.

Les résultats obtenus lors de l'émission de radio, des reportages et du journal télévisé concernant la production orale ont été:

D'abord, les apprenants se sentaient plus à l'aise lors des conversations et aussi lorsqu'ils devaient réaliser des entretiens. Ils ont appris un nouveau lexique qui leur a permis d'entretenir des conversations et des dialogues avec un public différent aÌ celui de la salle de classe. Ils ont acquis une certaine spontanéité puisqu'ils ont commenceì al participer beaucoup dans les cours de français et ils se montraient plus critiques par rapport à certaines situations, par exemple : ils prenaient beaucoup plus la parole en public et leurs arguments par rapport à quelques sujets d'actualités étaient plus élaborés.

Par ailleurs, ils étaient plus cohérents lorsqu'ils parlaient en français. Leurs idées étaient beaucoup mieux comprises et leur syntaxe était plus claire. Les phrases qu'ils faisaient au début des cours n'avaient aucune comparaison avec ce qu'ils faisaient après la mise en oeuvre des projets. Ces dernières avaient plus de sens et l'ordre des mots était intelligible.

De plus, quelques exemples qui permettront de constater l'évolution des apprenants seront illustrés: leurs problèmes au début seront mis en évidence ainsi que le constat à la fin des projets.

Avant la mise en œuvre des projets:

Situation de communication:

Bonjour sil. en quoi je peux vous aider?

\section{6}


Je viens de demander//s'il est possible de changer de famille d'accueil

Vous avez de difficulteìs avec la famille dans laquelle vous vivrell?

$\mathrm{h}$ (1.7) Oui, «en riant» je ne me sens bien vivant avec ils

Pourquoi vous ne vous sentez pas bien//?

Parce qu'il n'y a: de chaffage dans ma chambre

Et puis votre arriveì, sil. ils n'ont pas chaffage votre chambre?

Depuis j'ai commenceì habiter dans la maison

Oui, c'est deilicat (1.5) et vous avez une autre raison pourquoi vous voluez changer la famille d'accueil? ::: Oui, personne ne me parle, ils m'ignorent complèttement

J'imagine que c'a; (dort etre treÌs mal) à̀ l'aise pour vous

Oui, c'est vrai. je me sens mal à̀ l'aise

Eh bien, mademoiselle, j'infomerai immeìdiatement au comiteì d'eìcole de langues et dans deux jours vous pourrez passer.

Dans l'exemple citeì ci-dessus, on peut remarquer que tout au début les étudiants avaient beaucoup de problèmes concernant la syntaxe, les verbes étaient mal conjugués, ce qui empêchait la compréhension et le lexique était restreint. De plus, c'est grâce al̀ l'aide de ces situations qu'on a commenceì al identifier les erreurs qu'ils commettaient entre le passage de la graphie à̀ la phonie.

ApreÌs la mise en œuvre:

On voit bien qu'après la mise en œuvre des projets, ils avaient toujours quelques problèmes concernant la syntaxe, les fautes d'orthographe et les interférences. Cependant, il y eu plus de sens dans ce qu'ils disaient, et ils s'amélioraient jour après jour.

Exemple:

Qu'est-ce que vous pensez de la mode en France?

- Bien, d'abord je ne suis pas d'accord avec la mode en France parce qu'il y a quelques situations ouİ la majoriteì des femmes portent vetements avec la peau des animaux, en plus en France les personnages sont trel̀s vaniteux et les jeunes sont minces aÌ cause de la mode. 
- Je pense que, elle est trè̀s eilaborè̀e et parfait parce que nous connaissons la personne qui fait la mode que sont trè̀s connu comme Marcel Oran.h: ou Dior.

Quelle image croyez-vous que les colombiens projettent dans le monde?

- Bien :: je pense qui autour du monde, nous les colombiens nous avons beaucoup des images parce que bien, il y a beaucoup des pays ils consideÌrent comme des terroristes (1.5) comme des personnes terribles qui faisons la guerre. L'opinion est que la vision du monde est totalement diffeìrente à des nous trouvons ici Je pense que tout le monde, je ne pense pas qui ne sommes pas de terroristes, je pense qu'il y a quelques exemples mais la majoriteì des pays ils doivent changer le... sur notre pays.

Pourquoi croyez-vous que la France c'est connue?

Je pense que d'abord la langue parce que nous connaissons la France parce que nous eìcoutons parler de la langue franc'aise, je pense que le parfume parce que tout le monde parle du parfume qui sont produit en France et qui sont trel̀s fameuses $(t)$ Bien, je pense que la France c'est connue parce que nous avons eìcouteì du chocolat franc'ais, ce sont des chocolateìs treÌs fameuses pour le bon qu'ils sont ::: et peut-etre par exemple quand on eìcoute la radio, quand on eìcoute, je ne sais pas; les diffeìrents medias, on peut savoir on peut connaitre le preìsident ou nous avons eìcouteì le preìsident qu'il y avait le preìsident Sarkozy je pense que c'a c'est la part de la connaissance que nous avons de la France.

On peut prouver grâce aux situations citées préalablement qu'ils ont amélioré leur syntaxe. Les phrases qu'ils ont construites étaient plus cohérentes, le langage utiliseì était plus contextualiseì et porteur de sens; tout cela en raison de la connaissance préalable du contexte et parfois du lexique; mais les problèmes de phonème et graphie ont été récurrents.

Ainsi, la production orale est devenue l'une des activités langagières la plus développée par l'ensemble des apprenants. De même, ils sont arrivés à s'exprimer avec une grande faciliteì et ils sont arrivés al̀ accomplir certaines tâches qui les obligeaient al avoir une bonne production discursive.

Désormais, ils possèdent une compétence fonctionnelle en langue étrangère qui peut les aider à avancer dans l'amélioration de la production orale en FLE. De plus, ils ont amélioreì leur prononciation et bien qu'il existe encore des lacunes, ce sont des apprenants avec une motivation extraordinaire qui vont surmonter n'importe quel type de difficulté tout au long de l'apprentissage de cette langue.

\section{8}




\section{Conclusions}

Les progrès mentionnés ci-dessus ont été obtenus à̀ la fois grâce aux trois projets mis en œuvre et surtout au travail avec des outils technologiques qui ont provoqueì un déclic de l'activité langagière chez ces apprenants. C'est ainsi qu'au travers la réalisation de ces trois projets, des compétences linguistiques, sociolinguistiques et discursives ont été atteintes. Par ailleurs, les étudiants ont perçu le travail autrement, ils ont pris conscience de leur responsabilité dans la réalisation et la réussite des projets.

Il est important aussi de conclure sur le fait que travailler en pédagogie du projet aide beaucoup à pallier le manque d'autonomie et de collaboration chez les apprenants. À la fin du travail, les étudiants étaient plus autonomes, surtout lors de la réalisation d'activités en dehors de la salle de classe. Donc, si l'on continue al travailler dans cet esprit dans le système éducatif colombien et dans le cadre de l'enseignement du FLE, on pourrait avoir des résultats très positifs voire extraordinaires.

Ainsi la pédagogie de projet est non seulement une bonne méthode pour développer des compétences diverses, mais encore, c'est aussi un outil efficace pour les enseignants en langues qui peuvent ainsi aider les apprenants à surmonter des difficultés. Cette méthode

pourrait devenir un outil d'enseignement permanent dans les salles de classe et elle pourrait être introduite pour d'autres activités.

La pédagogie du projet change la manière traditionnelle d'enseigner, laissant la place au développement d'activités dans lesquelles les étudiants peuvent communiquer, se ressembler, échanger leurs avis et surtout favoriser l'ouverture de la classe au monde extérieur.

\section{Références Bibliographique}

Barbier, J.-M. (1991). Élaboration des projets d'action et planification, Paris: PUF.

Bordallo I., Ginestet J.-P. (1993). Pour une pédagogie du projet, Paris: Hachette.

Oxford, R. (1990). Language learning strategies: what every teacher should know, Boston.

Perrenoud, P. (2002). «Apprendre à l'école à travers des projets: Pourquoi? Comment?». En revista in Éducateur, № 14, pp. 6-11. 
Puren C., Bertocchini P., Costanzo E. (1998). Se former en didactique des langues. Paris: Ellipses.

Savignon, SJ. (1983). Communicative competence: theory and classroom practice.

Toffoli, D. (2008). Le plaisir de communiquer. IBIS ROUGE. Paris.

\section{Sitographie}

Guillén, Diaz C. (2003). Une exploration du concept «lexiculture» au sein de la Didactique des Langues-Cultures (p.p 109-110-111) http://revistas.ucm.es/edu/11300531/ articulos/DIDA0303110105A.PDF.

Groux, D. (2009). «Approches comparés de l'enseignement des langues et de la formation d'enseignants».

http://books.google.fr/books?id=Kymz9Udp1zQC\&pg=PA187\&lpg=PA187\&dq= dominique+groux +FLS 